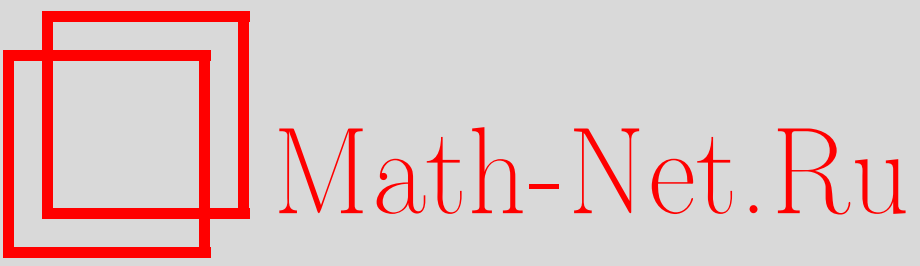

Н. Д. МакКай, Интегрируемость нелинейных сигма-моделей с границей, ТМФ, 2005, том 142, номер 2, 322-328

DOI: https://doi.org/10.4213/tmf1785

Использование Общероссийского математического портала Math-Net.Ru подразумевает, что вы прочитали и согласны с пользовательским соглашением

http://www.mathnet.ru/rus/agreement

Параметры загрузки:

IP : 3.85 .73 .92

26 апреля 2023 г., 14:51:21 
ТЕОРЕТИЧЕСКАЯ

И МАТЕМАТИЧЕСКАЯ

ФИЗИКА

Том 142, № 2

февраль, 2005

(C) 2005 г.

Н. Дж. МакКай*

\section{ИНТЕГРИРУЕМОСТЬ НЕЛИНЕЙНЫХ СИГМА-МОДЕЛЕЙ С ГРАНИЦЕЙ}

Приведены результаты по классической интегрируемости главной киральной модели и общих сигма-моделей с границами в (компактных) симметрических пространстваx.

Ключевые слова: интегрируемая теория поля, сигма-модели, интегрируемость теорий с границей.

\section{1. ВВЕДЕНИЕ}

C начала 1990-х годов параллельно с "революцией D-бран" в теории струн большое внимание было посвящено интегрируемым моделям с границей (работы [1] содержат ряд плодотворных идей, а [2] представляют собой ранние обзоры). Вместо того чтобы испортить "хорошие" алгебраические и аналитические свойства объемной интегрируемости, аккуратный выбор граничного условия может не только не нарушить интегрируемости, а привести к элегантным дополнительным структурам.

Однако внимание, посвяшенное нелинейной сигма-модели (т.е. гармоническим отображениям из полуплоскости), было относительно невелико и ограничивалось в основном анализом $O(N)$-модели (модели на $S O(N) / S O(N-1)$ ) [3]-[6]. Здесь мы представляем работу, начинающуюся с рассмотрения главного кирального поля (на компактной группе Ли $G$ ) [7], [8], которое затем распространяется на компактные симметрические пространства $G / H$ (с главным киральным полем, реализованным как симметрическое пространство $G \times G / G)[9]$.

\section{2. ГЛАВНОЕ КИРАЛЬНОЕ ПОЛЕ}

Главное киральное поле определяется плотностью лагранжиана

$$
\mathcal{L}=-\frac{1}{2}\left\langle g^{-1} \partial_{\mu} g, g^{-1} \partial^{\mu} g\right\rangle,
$$

*Department of Mathematics, University of York, UK. E-mail: nm15@york.ac.uk 
где определенное на пространстве Минковского при $-\infty<x \leqslant 0$ поле $g(t, x)$ принимает значения в компактной классической группе Ли $G$ и $\langle\cdot, \cdot\rangle$ - отрицательно-определенное инвариантное скалярное произведение на алгебре Ли $\mathfrak{g}$ этой группы. Граничное уравнение движения имеет вид

$$
\left\langle g^{-1} \partial_{1} g, g^{-1} \delta g\right\rangle=0 \quad \text { при } x=0,
$$

где вариация берется по всем $\delta g$ таким, что $g^{-1} \delta g \in \mathfrak{g}$. Ясно, что граничные условия Неймана $\partial_{1} g=0$ или Дирихле $\delta g=0$ удовлетворяют этому уравнению. Однако ему удовлетворяют также и два типа смешанных условий,

$$
\begin{aligned}
& g(t, 0) \in g_{L} H g_{R}^{-1}, \\
& g(t, 0) \in g_{L} \frac{G}{H_{\alpha}} g_{R}^{-1},
\end{aligned}
$$

где $G / H_{\alpha} \cong\left\{\alpha(g) g^{-1} \mid g \in G\right\}$ - вложение Картана $G / H_{\alpha}$ в $G, H_{\alpha}$ - инвариантная относительно инволютивного (т.е. удовлетворяющего условию $\alpha^{2}=1$ ) автоморфизма $\alpha^{1}$ ) подгруппа группы $G, g_{L}, g_{R}$ - фиксированные элементы группы $G$. (В действительности нужно соблюдать известную осторожность: вложение Картана является локальным диффеоморфизмом, однако, как показано в [10], может не быть (в конечной части) ни взаимно однозначным отображением, ни отображением на.)

Чтобы убедиться в этом, определим сохраняющиеся токи со значениями в алгебре $\mathfrak{g}$, которые порождают $G \times G$ симметрию $g \mapsto U g V^{-1}$, как

$$
j_{\mu}^{L}=\partial_{\mu} g g^{-1}, \quad j_{\mu}^{R}=-g^{-1} \partial_{\mu} g,
$$

и заметим, что граничное уравнение движения эквивалентно условию $\left\langle j_{0}, j_{1}\right\rangle=0$ или, в компонентах светового конуса, $\left\langle j_{+}, j_{-}\right\rangle=0$ (в силу цикличности следа неважно, используется ли $j^{L}$ или $j^{R}$ ). Наше киральное граничное условие эквивалентно условиям

$$
g_{L}^{-1} j_{0}^{L} g_{L}=\alpha\left(g_{L}^{-1} j_{0}^{L} g_{L}\right) \in \mathfrak{h}_{\alpha}, \quad g_{R}^{-1} j_{0}^{L} g_{R}=\alpha\left(g_{R}^{-1} j_{0}^{L} g_{R}\right) \in \mathfrak{h}_{\alpha} \quad \text { при } x=0,
$$

где $\mathfrak{g}=\mathfrak{h}_{\alpha} \oplus \mathfrak{m}_{\alpha} ; \mathfrak{h}_{\alpha}$ порождает $H_{\alpha}$ и является собственным пространством с собственным значением +1 , в то время как $\mathfrak{m}_{\alpha}$ является собственным пространством с собственным значением -1 . Граничное уравнение движения требует в таком случае, чтобы пространственные компоненты принимали значения в $\mathfrak{m}_{\alpha}$. Все вместе это дает

$$
g_{L}^{-1} j_{+}^{L} g_{L}=\alpha\left(g_{L}^{-1} j_{-}^{L} g_{L}\right) \quad \text { при } \quad x=0
$$

и аналогичную формулу для $j^{R}$. Аналогично можно показать, что некиральное граничное условие $(3)$ влечет $g_{L}^{-1} j_{0}^{L} g_{L}=\alpha\left(g_{R}^{-1} j_{0}^{R} g_{R}\right)$, и, таким образом, необходимо

$$
g_{L}^{-1} j_{+}^{L} g_{L}=\alpha\left(g_{R}^{-1} j_{-}^{R} g_{R}\right) \quad \text { при } \quad x=0 .
$$

\footnotetext{
${ }^{1)}$ Впредь мы будем называть такие отображения инволюциями.
} 
Для построения сохраняюшихся коммутируюших локальных зарядов модели используются некоторые специальные способы выбора инвариантных тензоров [11]. То, что локальные заряды при наличии этих граничных условий остаются сохраняюшимися и коммутируюшими на полупрямой, следует из простого поведения этих тензоров под действием преобразования $\alpha$. Если $\alpha$ является внутренним автоморфизмом, то тензоры являются, конечно, инвариантными относительно $\alpha$, однако если $\alpha$ является внешним автоморфизмом, то они могут таковыми и не быть. На самом деле, они всегда имеют собственные значения \pm 1 относительно $\alpha$ [12], и этого достаточно, чтобы обеспечить возможность построения одного сохраняюшегося заряда из каждой пары локальных зарядов со спинами \pm 1 (например, энергии, но не импульса). Это, а также совместимость скобок Пуассона с рассматриваемыми граничными условиями, детально описаны в [7].

В дополнение к локальным модель имеет нелокальные сохраняющиеся заряды. В объемной модели $G_{L} \times G_{R}$ симметрия, порождаемая токами $j^{L}$ и $j^{R}$, находится внутри большей $Y(\mathfrak{g}) \times Y(\mathfrak{g})$ симметрии, где $Y(\mathfrak{g})$ - алгебра янгианов. Эта симметрия порождается зарядами

$$
\begin{aligned}
Q^{(0) a} & =\int j_{0}^{a} d x \\
Q^{(1) a} & =\int j_{1}^{a} d x-\frac{1}{2} f^{a} b c \int j_{0}^{b}(x) \int^{x} j_{0}^{c}(y) d y d x,
\end{aligned}
$$

используюшими, соответственно, токи $j^{L}$ и $j^{R}$, представленные в виде разложения $j=$ $j^{a} t_{a}$, где $t^{a}$ - генераторы алгебры $\mathfrak{g},\left[t_{a}, t_{b}\right]=f_{a b}{ }^{c} t_{c}$. Для объемной модели интегрирование ведется по всей прямой $(-\infty, \infty)$. Однако на полупрямой $(-\infty, 0]$ эти заряды, вообще говоря, более не сохраняются. Тем не менее существуют два важных набора зарядов, которые остаются (классически) сохраняюшимися. Используем буквы $i, j, k, \ldots$ для записи $\mathfrak{h}_{\alpha}$-индексов и $p, q, r, \ldots$ для $\mathfrak{m}_{\alpha}$-индексов и заметим, что единственными не обращающимися в нуль структурными константами являются $f^{i}{ }_{j k}$ и $f^{i}{ }_{p q}$ (и их циклические перестановки); тогда, как показано в [8], искомыми зарядами будут

$$
\begin{aligned}
& Q^{(0) i}, \\
& \widetilde{Q}^{(1) p} \equiv Q^{(1) p}+\frac{1}{2} f^{p}{ }_{q i} Q^{(0) i} Q^{(0) q} .
\end{aligned}
$$

Первое множество порождает $H_{\alpha}$, в то время как второе представляет собой некоторый остаток нелокальных зарядов. В предположении, что второе множество зарядов выдерживает квантование, оно может быть записано в виде

$$
\widetilde{Q}^{(1) p}=Q^{(1) p}+\frac{1}{4}\left[C_{2}^{\mathfrak{h}}, Q^{(0) p}\right],
$$

где $C_{2}^{\mathfrak{h}} \equiv \gamma_{i j} Q^{(0) i} Q^{(0) j}$ - сужение квадратичного оператора Казимира алгебры $\mathfrak{g}$ на $\mathfrak{h}_{\alpha}, \gamma_{i j}=f_{i a}{ }^{b} f_{j b}{ }^{a} \quad(\hbar=1)$. Сохранение этих зарядов может быть далее использовано для определения $S$-матриц моделей с границами с точностью до обшего скалярного 
множителя. Метод основан на том, что, во-первых, из сохранения заряда $Q^{(0) i}$ следует тривиальность действия матрицы отражения на неприводимых представлениях $H_{\alpha}$, а во-вторых, сохранение зарядов $\widetilde{Q}^{(1) p}$ определяет относительные значения коэффициентов этих проекторов в терминах значений оператора $C_{2}^{\mathfrak{h}}$. Подробности можно найти в работе [8] и для всех $\mathfrak{g}$ в работе [13], из которых видно, что состоятельность рассматриваемого подхода обусловлена "теоремой о симметрических пространствах" из [14].

Подобно тому как алгебра $Y(\mathfrak{g})$ может рассматриваться как деформация полиномиальной алгебры $\mathfrak{g}[z]\left(\right.$ с $\left.Q_{1}^{a}=z Q_{0}^{a}\right)$, на алгебру, порожденную зарядами $Q^{(0) i}$ и $\widetilde{Q}^{(1) p}$, можно смотреть как на инвариант деформации подалгебры "твистованных" полиномов в $\mathfrak{g}[z]$ относительно совместного действия $\sigma$ и $z \mapsto-z$. По этой причине она известна как алгебра "твистованных янгианов", и мы обозначаем ее $Y\left(\mathfrak{g}, \mathfrak{h}_{\alpha}\right)$. Эта алгебра хорошо известна и изучена в литературе (см., например, [15]), хотя и не в обшей форме.

\section{3. СИГМА-МОДЕЛИ В СИММЕТРИЧЕСКИХ ПРОСТРАНСТВАХ}

Объемная модель на общем компактном симметрическом пространстве $G$ / $H$ подробно описана в работах [10], [16]. Заметим, что необходимо отличать инволюцию, которая определяет пространство $G / H$ (мы будем использовать для нее обозначение $\sigma$ ), от инволюции, которая появляется в граничных условиях. По этой причине мы были внимательны и использовали индекс $\alpha$ везде в предыдущем разделе.

Коротко говоря, калибровка инварианта подгруппы $H$ относительно $\sigma$ достигается требованием локальной симметрии модели $g(t, x) \mapsto g(t, x) h(t, x)$. Чтобы достигнуть этого, введем калибровочное поле $A_{\mu}(t, x) \in \mathfrak{h}$ и ковариантную производную $D_{\mu} g \equiv$ $\delta_{\mu} g-g A_{\mu}$. Тогда плотность лагранжиана имеет вид

$$
\mathcal{L}=-\frac{1}{2}\left\langle J_{R \mu}, J_{R}{ }^{\mu}\right\rangle=-\frac{1}{2}\left\langle J_{L \mu}, J_{L}{ }^{\mu}\right\rangle=-\frac{1}{8}\left\langle q^{-1} \partial_{\mu} q, q^{-1} \partial^{\mu} q\right\rangle
$$

Ток

$$
J_{R}^{\mu} \equiv g^{-1} D^{\mu} g=j^{\mu}-A^{\mu},
$$

связанный с локальной правой $H$-симметрией, является калибровочно-ковариантным, и уравнения движения суть $D_{\mu} J_{R}^{\mu}=0$, тогда как уравнения движения для $A_{\mu}$ накладывают ограничение $J_{R}^{\mu}=0$ на $\mathfrak{h}$. Другой способ состоит в том, чтобы выразить плотность лагранжиана $\mathcal{L}$ в терминах калибровочно-инвариантного тока

$$
J_{L}^{\mu} \equiv-\left(D^{\mu} g\right) g^{-1},
$$

который (нековариантно) сохраняется, $\partial_{\mu} J_{L}^{\mu}=0$. Существует также описание модели исключительно в терминах (калибровочно-инвариантного) поля $q=\sigma(g) g^{-1}$ такого, что $q(t, x)$ - поле, принимающее значения во вложении Картана; в этом случае $J_{L}^{\mu}=$ $q^{-1} \partial^{\mu} q / 2$. 
Уравнения движения позволяют построить локальные сохраняющиеся заряды, плотности которых являются полиномами по $J_{R}^{\mu}$ или $J_{L}^{\mu}[12]$. Можно подумать, что инвариантная формулировка удобнее для работы, но в ней труднее оперировать скобками Пуассона [17], чем при использовании калибровочной формулировки. Нелокальные заряды могут быть построены обычным способом из $2 J_{L}^{\mu}$; они порождают алгебру $Y(\mathfrak{g})$.

Отступая от наших рассуждений, отметим, что различные аспекты объемной квантовой $G / H$-модели все еще не вполне поняты. Сушествуют потенциальные аномалии для непростых групп $H$ [18], [19], и даже для простых групп $H$ нет общего описания характера спектра. Частицы должны образовывать $Y(\mathfrak{g})$-мультиплеты, но какие именно, предстоит определить на основании допустимых значений локальных зарядов, которые должны быть связаны с системами корней пространства $G / H$ [20].

Поскольку здесь мы не собираемся вдаваться в детали канонического формализма, зададим граничные условия, используя калибровочно-инвариантный ток $J_{L}^{\mu}$, в терминах которого граничное уравнение движения может быть записано как

$$
\left\langle J_{L}^{+}, J_{L}^{-}\right\rangle=0
$$

Этому уравнению удовлетворяет $g(t, 0)=g_{0} l(t)$ с фиксированным $g_{0}$ и $l \in H_{\tau}$, где $H_{\tau}-$ инвариант подгруппы относительно некоторой дополнительной инволюции $\tau$. Основной доказанный в работе [9] результат состоит в том, что для совместимости со скобками Пуассона и для сохранения локальных зарядов эта инволюция должна коммутировать с инволюцией $\sigma$. В таком случае условие склеивания для токов, аналогичное $(6),(7)$, имеет вид

$$
\tau\left(g_{0}^{-1} J_{L}^{+} g_{0}\right)=g_{0}^{-1} J_{L}^{-} g_{0}
$$

Тривиальные случаи $\tau=1$ и $\tau=\sigma$ отвечают чистым граничным условиям Неймана и Дирихле, соответственно.

Первый естественный пример - это пример главного кирального поля, реализованного как симметрическое пространство $G \times G / G_{\text {diagonal }}$, где $G_{\text {diagonal }}=\{(g, g) \mid g \in G\}$ (подробности приведены в [9]). Суть в том, что когда $\sigma$ - оператор перестановки в $G \times$ $G$, единственными допустимыми нетривиальными $\tau$ являются $(\alpha, \alpha)$ и $\sigma(\alpha, \alpha)$ (где $\alpha-$ произвольно выбранная инволюция), и это приводит к двум типам граничных условий $(2),(6)$ и $(3),(7)$, соответственно.

Следуюший пример, также содержашийся в [9], есть $S O(3) / S O(2)$; некоторые подробные результаты для этого примера имеются в [3], [6]. Если допустить, что $\tau$ - произвольная инволюция, и потому $H_{\tau}$ - произвольная $S O(2)^{\prime}$ группа, то это даст D-подмногообразие $\left\{\sigma\left(g_{0} l\right)\left(g_{0} l\right)^{-1} \mid l \in S O(2)^{\prime}\right\} \subset S^{2}$, которое есть произвольная окружность. Если потребовать, чтобы $\tau$ и $\sigma$ коммутировали, то эта окружность становится окружностью большого круга.

Нетрудно, по крайней мере для классических групп $G$, составить список других примеров пар коммутирующих инволюций. Например, в грассмановом случае $S U(N) /$ $S(U(N-M) \times S U(M)), S O(N) / S O(N-M) \times S O(M)$ или $S p(2 n) / S p(2 n-2 m) \times S p(2 m)$; 
для любого другого $M^{\prime}$ существует группа $S U\left(N-M^{\prime}\right) \times S U\left(M^{\prime}\right)(S O, S p)$, определяющая инволюция которой коммутирует с $\sigma$. Поэтому для $\mathbb{C} P^{N-1}\left(S^{N-1}\right)$-модели находим, что $\mathbb{C} P^{M^{\prime}-1}\left(S^{M^{\prime}-1}\right.$, см. [5]) есть допустимое (нетривиальное) D-многообразие для всех $M^{\prime}=2, \ldots, N-1$.

\section{4. ЗАМЕЧАНИЯ ОТНОСИТЕЛЬНО КВАНТОВЫХ МОДЕЛЕЙ}

Программа исследования квантовой модели для главного кирального поля в известной степени не содержит трудностей. Локальные [18], [21] и нелокальные [22] заряды выдерживают квантование и согласуются с предложенными точными $S$-матрицами объемной модели [23] и модели с границей [7]. Остается только достичь полного понимания в вопросах того, как устроен спектр модели с границей и его взаимодействия.

Для обшего случая моделей в симметрическом пространстве мы, однако, далеки от такого положения дел. Как было сказано выше, в настояшее время сушествует убеждение, что объемные модели не являются интегрируемыми для непростых групп $H$, хотя результаты последнего времени снова возвращают нас к этому вопросу [24]: кажется вероятным, что, по крайней мере, некоторые такие модели являются интегрируемыми в квантовом случае. Даже для тех объемных моделей, которые непременно являются таковыми, не существует общего описания характера их спектра, имеющего столь же элегантный вид, как для главного кирального поля [25]. Для моделей в симметрическом пространстве с границей мы имеем теперь, по крайней мере, общую постановку проблемы их классической интегрируемости. Квантовые модели с границей имеют $Y\left(\mathfrak{g}, \mathfrak{h}_{\tau}\right)$-симметрию там, где они сушествуют, и поэтому для них можно использовать известные решения уравнения отражения. Однако первоочередная задача состоит в том, чтобы установить условия интегрируемости квантовой модели, возможно, посредством распространения на общий случай $G / H$ граничного варианта [5] техники исчисления аномалий для локальных зарядов [18].

Благодарности. Автор благодарен Королевскому обществу за грант на оплату транспортных расходов и организаторам конференции за гостеприимство.

\section{Список литературы}

[1] И. В. Чередник. ТМФ. 1984. Т. 61. № 1. С. 35; S. Ghoshal, A. Zamolodchikov. Int. J. Mod. Phys. A. 1994. V. 9. P. 3841; Erratum. V. 9. P. 4353; hep-th/9306002.

[2] E. Corrigan. Reflections. hep-th/9601055; Integrable field theory with boundary conditions. In: Frontiers in Quantum Field Theory. Eds. Chao-Zheng Zha, Ke Wu. Singapore: World Scientific, 1998. P. 9; hep-th/9612138.

[3] E. Corrigan, Z.-M. Sheng. Int. J. Mod. Phys. A. 1997. V. 12. P. 2825; hep-th/9612150.

[4] S. Ghoshal. Phys. Lett. B. 1994. V. 334. P. 363; hep-th/9401008.

[5] M. Moriconi. Nucl. Phys. B. 2001. V. 619. P. 396; hep-th/0108039; Integrable boundary conditions for the $O(N)$ nonlinear sigma model. In: Statistical Field Theories. Proc. of the NATO Adv. Res. Working. Como, Italy, 18-23 June, 2001. Part II. NATO Sci. Ser. II. Math. Phys. Chem. V. 73. Eds. A. Capelli, G. Mussardo. Dordrecht: Kluwer, 2002; hep-th/0111195.

[6] W. He, L. Zhao. Phys. Lett. B. 2003. V. 570. P. 251; hep-th/0307002. 
[7] N. MacKay, B. Short. Commun. Math. Phys. 2003. V. 233. P. 313; Erratum. 2004. V. 245. P. 401; hep-th/0104212.

[8] G. Delius, N. MacKay, B. Short. Phys. Lett. B. 2001. V. 522. P. 335; Erratum. 2002. V. 524. P. 401; hep-th/0109115.

[9] N. MacKay, C. A.S. Young. Phys. Lett. B. 2004. V. 588. P. 221; hep-th/0402182.

[10] H. Eichenherr. Nucl. Phys. B. 1980. V. 164. P. 528; 1987. V. 282. P. 745; J.-P. Antoine, B. Piette. J. Math. Phys. 1987. V. 28. P. 2753.

[11] J. M. Evans, M. Hassan, N. J. MacKay, A. Mountain. Nucl. Phys. B. 1999. V. 561. P. 385; hep-th/9902008.

[12] J. M. Evans, A. J. Mountain. Phys. Lett. B. 2000. V. 483. P. 290; hep-th/0003264.

[13] N. MacKay. J. Phys. A. 2002. V. 35. P. 7865; math.QA/0205155.

[14] P. Goddard, W. Nahm, D. Olive. Phys. Lett. B. 1985. V. 160. P. 111; C. Daboul. J. Math. Phys. 1996. V. 37. P. 3576; hep-th/9604108.

[15] А. Молев, М. Назаров, Г. Ольшанский. УМН. 1996. Т. 51. № 2. С. 27.

[16] H. Eichenherr, M. Forger. Nucl. Phys. B. 1978. V. 155. P. 381.

[17] M. Forger, J. Laartz, U. Schaeper. Commun. Math. Phys. 1992. V. 146. P. 397; hep-th/9201025.

[18] Y. Y. Goldschmidt, E. Witten. Phys. Lett. B. 1980. V. 91. P. 392.

[19] E. Abdalla, M. C. B. Abdalla. Phys. Rev. D. 1981. V. 23. P. 1800; E. Abdalla, M. Gomes, M. Forger. Nucl. Phys. B. 1982. V. 210. P. 181.

[20] J. M. Evans. Nucl. Phys. B. 2001. V. 608. P. 591; hep-th/0101231.

[21] J.M. Evans, M. Hassan, N. J. MacKay, A.J. Mountain. Conserved charges and supersymmetry in principal chiral models. hep-th/9711140.

[22] D. Bernard. Commun. Math. Phys. 1991. V. 137. P. 191.

[23] E. Ogievetsky, N. Reshetikhin, P. Wiegmann. Nucl. Phys. B. 1987. V. 280. P. 45; E. Ogievetsky, P. Wiegmann. Phys. Lett. B. 1986. V. 168. P. 360.

[24] P. Fendley. Phys. Rev. B. 2001. V. 63. P. 104429; cond-mat/0008372; JHEP. 2001. V. 0105. P. 050; Integrable sigma models and perturbed coset models. hep-th/0101034; A. Babichenko. Phys. Lett. B. 2003. V. 554. P. 96; hep-th/0211114.

[25] P. E. Dorey. Nucl. Phys. B. 1991. V. 358. P. 654. 